Original Paper

http://indexmedicus.afro.who.int

\title{
Les Loranthaceae, parasites des arbres et arbustes : cas du département de Katiola, au nord de la Côte d'Ivoire
}

\author{
Armèle Affoué KOFFI ${ }^{1 *}$, Faustine Akossoua KOUASSI ${ }^{2}$ et \\ Serge Beranger Koua N'GORAN ${ }^{2}$ et Dodiomon SORO ${ }^{1}$ \\ ${ }^{1}$ Laboratoire de Botanique, U.F.R. Biosciences Université Félix Houphouët Boigny, \\ Abidjan, 22 BP 582 Abidjan 22, Côte d'Ivoire. \\ ${ }^{2}$ Centre National de Floristique Université Félix Houphouët Boigny, \\ Abidjan, 22 BP 582 Abidjan 22, Côte d'Ivoire. \\ *Auteur correspondant, E-mail : beugrearmele@gmail.com ; Tel : 0022507575282
}

\section{RE்SUME்}

Les Loranthaceae sont des plantes parasites qui causent de nombreux dommages sur les arbres et les arbustes se traduisant par l'envahissement et la défoliation des plantes infestées. Dans le but d'identifier et de préciser l'infestation des arbres et arbustes par les Loranthaceae, une étude botanique a été réalisée en zone de savane, plus précisément au Nord de la Côte d'Ivoire dans le département de Katiola. La méthode itinérante a été utilisée pour inventorier les arbres et les arbustes infestés par ces parasites et identifier toutes les espèces de Loranthaceae parasites présentes. Au total, deux espèces de Loranthaceae ou parasites des plantes vasculaires ont été dénombré. Ce sont: Agelanthus dodoneifolius et Tapinanthus bangwensis. L'analyse des données obtenues montre un taux de parasitage de 0 p.c. au niveau des cultures pérennes. Les taux de parasitage des arbres et des arbustes, dans la végétation naturelle ou spontanée sont respectivement de 6,33 p.c et 3,51p.c. Le test de $\mathrm{Khi}^{2}$ utilisé pour comparer les taux de parasitage des arbres et arbustes montre qu'il n'y a pas de différence significative entre les arbres et les arbustes infestés.

(c) 2014 International Formulae Group. All rights reserved.

Mots clés : Taux de parasitage, plante vasculaire, végétation spontanée.

\section{INTRODUCTION}

Les Loranthaceae sont des plantes vasculaires parasites des végétaux; elles affectent négativement la croissance et la valeur économique des plantes hôtes qui finissent par mourir (Boussim, 2002). Ces parasites vasculaires sont présents dans toutes les régions intertropicales et dans certaines zones tempérées. En Afrique, dans des pays tels que le Burkina faso, le Cameroun, le Gabon, le Ghana, le Mali et bien d'autres, les Loranthaceae causent d'importants dégâts dans les formations naturelles et les plantations (Boussim, 2002; Boussim et Nayéré, 2004). En Côte d'Ivoire, plusieurs études ont été réalisées sur les Loranthaceae, en particulier les travaux de Traoré et al., (2003). Ces parasites, jadis ignorés par les structures de recherches ivoiriennes, constituent aujourd'hui un véritable fléau, vu les dégâts causés par ceux-ci aussi bien dans les formations végétales naturelles (Soro et al., 2006) que dans les plantations de culture de rente (Soro et al., 2006; Amon et al., 
2010). Ces plantes vasculaires en forme de petits arbrisseaux se développent sur le tronc et les branches de leur hôte. Elles détournent la sève brute de la plante hôte. La croissance de la plante hôte est alors ralentie et finit par s'estomper. Dans le département de Katiola, aucune étude scientifique sur les Loranthaceae parasites n'a été encore réalisée. Il s'avère donc impérieux de mener des études de reconnaissance de ces hémiparasites dans cette localité, afin d'entreprendre des actions de lutte contre les Loranthaceae. L'objectif général de cette étude est d'identifier et de localiser les différentes espèces de Loranthaceae, parasites des arbres et arbustes ; dans le département de Katiola. Spécifiquement, il s'agira : d'identifier toutes les espèces de Loranthaceae parasites des végétaux dans la zone d'étude; d'inventorier les arbres et arbustes hôtes de ces parasites vasculaires ; d'évaluer l'ampleur des attaques des Loranthaceae sur les arbres et arbustes de la localité.

\section{MATERIEL ET METHODES} Site de l'étude

L'étude a été menée dans le département de Katiola, dans le nord de la Côte d'Ivoire. Situé entre $5^{\circ} 06$ et $5^{\circ} 07$ de longitude Ouest et entre $8^{\circ} 08$ et $8^{\circ} 15$ de latitude Nord, le département de Katiola présente un climat de type subtropical. Il est caractérisé par la savane préforestière. La végétation est en général constituée de savanes arborées et de savanes herbeuses, parsemées d'ilots de forêts galeries surtout le long des cours d'eaux (Guillaumet et Adjanohoun, 1971).

Les sous-préfectures de Timbé, Fronan et Katiola ont été choisis pour abriter l'étude. Cet échantillonnage a permis de parcourir le maximum de formations végétales couvrant le département de Katiola.

\section{Collecte des données}

L'étude repose sur des observations faites sur le terrain. Elle a consisté à inventorier les Loranthaceae et leurs hôtes dans le département de Katiola. Plusieurs méthodes d'inventaire botanique existent en fonction des objectifs de chaque étude (Sinsin, 1993; Sokpon, 1995 ; Habiyaremye, 1997 ; Ganglo, 1999). Pour cette étude, nous nous sommes basés sur la méthode d'inventaire itinérant. Elle a consisté à parcourir dans différentes directions les sites retenus le long des pistes. Les formations végétales naturelles et les vergers ont été parcourus. Les arbres et arbustes spontanés situés de part et d'autres des pistes ont été observés. Les plantes hôtes parasitées ont été inventoriées ainsi que les espèces de Loranthaceae rencontrées. Au niveau des vergers, des parcelles unitaires d'un hectare ont été posés. Pour chaque arbre rencontré, nous avons noté plusieurs paramètres ; notamment la présence ou l'absence de parasites, le nom de l'espèce ou des espèces de Loranthaceae présentes, le nom de l'espèce hôte, le nombre d'arbres et d'arbuste infestés, et le nombre de touffes de parasites présents sur chaque arbre. Pour chaque parasite et chaque arbre hôte, nous avons prélevé un échantillon pour une confirmation au laboratoire de l'identification faite sur le terrain. La localisation du ou des parasites sur l'hôte a été aussi notée.

Des enquêtes ethnobotaniques basées sur des interrogations directes ont été effectuées de juillet à octobre 2007 dans les trois sous-préfectures choisies. Dans les villages, nous avons interrogé les populations riveraines qui sont en contact permanent avec les peuplements végétaux. Le questionnaire comportait des questions ouvertes. L'objectif visé, était de recenser toutes les espèces de Loranthaceae et les méthodes de lutte utilisées contre ces parasites.

\section{Analyse des données Analyse qualitative}

L'analyse qualitative (famille, genre, espèces) a été réalisée à partir de la liste floristique de toutes les espèces (Loranthaceae parasites et plantes hôtes) inventoriées. Les espèces énumérées ont été identifiées au Centre National de Floristique et par 
comparaison à celles énumérées par certains auteurs (Hévécam, 1995; Robbins et al., 1996 ; Koffi, 2004 ; Amon, 2006 ; Engone Obiang et Sallé, 2006 ; Soro, 2010).

\section{Analyse quantitative}

Le dénombrement des parasites et des plantes hôtes a permis de déterminer le taux de parasitage et l'intensité de parasitage des arbres et arbustes par les Loranthaceae.

taux de parasitage ou taux d'infestation (T), c'est le pourcentage de plants infestés par rapport au nombre total de plants observés:

$$
\mathrm{T}=(\mathrm{n} / \mathrm{N}) \times 100
$$

\section{$\mathrm{N}=$ Nombre total de plants observés} $\mathrm{n}=$ nombre de plants infestés

- Intensité de parasitage ou intensité d'infestation (I), elle exprime l'ampleur de l'infestation sur les individus parasités. Il est exprimé ici par le nombre moyen de touffes de Loranthaceae observées par individu :

$$
\mathbf{I}=\frac{\sum \mathrm{Ni}}{\mathrm{Nii}}
$$

$\mathrm{Ni}=$ nombre total de touffes de parasite

Nii $=$ nombre total de plants parasités

Un test de comparaison (Khi ${ }^{2}$ de Pierson) deux à deux des taux de parasitage a été réalisé à l'aide du logiciel Statistica (version 6.0).

\section{RESULTATS}

\section{Diversité floristique qualitative}

L'inventaire botanique a permis de recenser 2 espèces de Loranthaceae ; ce sont Tapinanthus bangwensis (Engl. et K. Krause) Danser et Agelanthus dodoneifolius (D.C.) Polh et Wiens dans la zone de l'étude.

Les arbres et arbustes hôtes rencontrées dans le département de Katiola sont: Ficus sciarophylla Warb. (Moraceae), Parkia biglobosa (Jacq.) R. Br. ex G. Don. (Mimosaceae), Vitellaria paradoxa C.F. Gaertn. (Sapotaceae), Terminalia avicennioides Guill \& Perr. (Combretaceae). Parinari curratellifolia Planch. ex Benth.
(Chrysobalanaceae) et Pilliostigma thonningii (Schum.) Milne-Redh. (Caesalpiniaceae). Ces espèces hôtes appartiennent à 6 genres reparties en 6 familles. Au niveau des cultures de rentes observées, aucune espèce de Loranthaceae parasite n'a été rencontrée sur les plants.

\section{Caractéristiques botaniques des Loranthaceae recensées}

Dans le département de Katiola, 2 espèces de Loranthaceae ont été inventoriées sur les arbres et arbustes. Agelanthus dodoneifolius est un arbuste très branchu avec des branches pouvant atteindre 1,5 $\mathrm{m}$ de long. La plante est glabre avec des rameaux légèrement anguleux ou aplati. Les feuilles sont alternes entières et opposées (Figure 1). Tapinanthus bangwensis est une plante glabre avec la branche principale atteignant à sa base $2,5 \mathrm{~cm}$ de diamètre. Les rameaux sont d'un brun-roussâtre, ou noirâtre, parfois pendants, atteignant jusqu'à $70 \mathrm{~cm}$. Ses organes jeunes sont lisses, puis couvert de petites lenticelles. Les feuilles simples, entières, sont opposées ou sub-opposées et à pétiole court. Le limbe est largement ovale elliptique (Figure 2).

\section{Caractérisation de l'infestation Taux d'infestation}

Le taux d'infestation est représenté à la Figure 3. La famille des Sapotaceae est la plus infestée, tandis que celle des Chrysobalanaceae est la moins parasitée. Nous n'avons pas rencontré de parasites sur les cultures arborescentes (manguier, anacarde) pratiquées dans cette région.

Les résultats obtenus après analyse des données montrent que le taux de parasitage est de 6,33 p.c. pour les arbres et de 3,51 p.c. pour les arbustes. Ce taux est de 5,47 p.c., pour l'ensemble des arbres et arbustes. Le test $\mathrm{Khi}^{2}$ réalisé pour comparer le pourcentage des arbres à celui des arbustes, est non significatif, autrement dit les arbres sont autant infestés 
que les arbustes dans le département de Katiola (Tableau 1).

\section{Intensité d'infestation}

L'intensité d'infestation ou le nombre moyen de parasite est de 4,3 touffes/plant pour les arbres et de 0,65 touffe/plant pour les arbustes. L'intensité d'infestation de l'ensemble des arbres et arbustes est de 5,52 touffes/plant.

$\mathrm{Du}$ point de vue épidémiologique, Agelanthus dodoneifolius est très fréquente et très envahissante. Elle a été observée sur près de 99,22 p.c. des arbres et arbustes infestées alors que Tapinanthus bangwensis ne parasite que moins de 1 p.c. des arbres et arbustes infestés. Agelanthus dodoneifolius et Tapinanthus bangwensis poussent en général à l'extrémité du houppier. Chez l'espèce Pilliostigma thonningii, qui est un arbuste, certaines touffes de Loranthaceae ont été observées à l'intérieur du houppier.
Dégâts des Loranthaceae sur les arbres et arbustes et méthodes de lutte utilisées par les populations locales

Les observations faites ont permis de noter certaines perturbations dues aux Loranthaceae sur les arbres et arbustes. Les perturbations les plus apparentes causées par les parasites sont : l'envahissement de l'individu parasité, la défoliation des pieds infestés particulièrement dans la zone attaquée, l'hypertrophie de la partie de la branche située en aval du point de fixation. Les enquêtes effectuées auprès des populations nous ont permis de constater qu'aucune méthode de lutte contre ces parasites n'est utilisée par les riverains. Selon eux, la présence des Loranthaceae sur les arbres et arbustes dans les végétations naturelles n'est pas nuisible, mais plutôt bénéfique pour la population qui les utilise pour le traitement de plusieurs maux. Cependant, la population estime que la méthode mécanique par suppression de la branche parasitée peut être utilisée aisément.

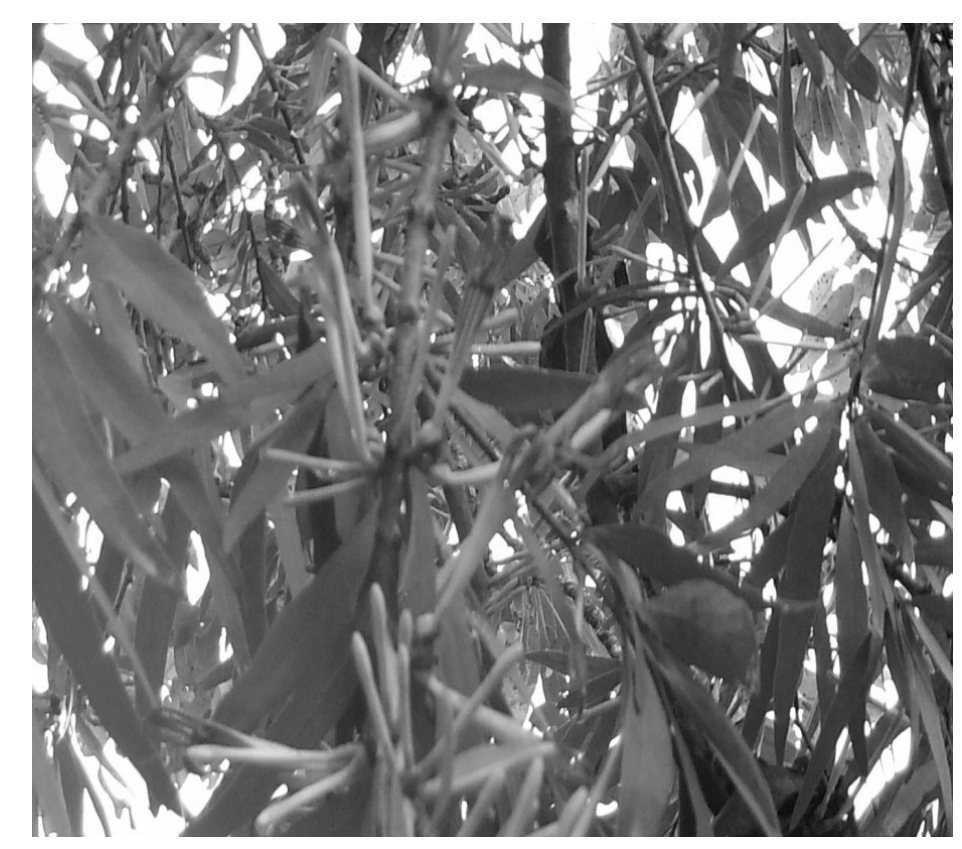

Figure 1: Rameaux feuillés et florifères de Agelanthus dodoneifolius parasite des arbres et arbustes dans le département de Katiola. 


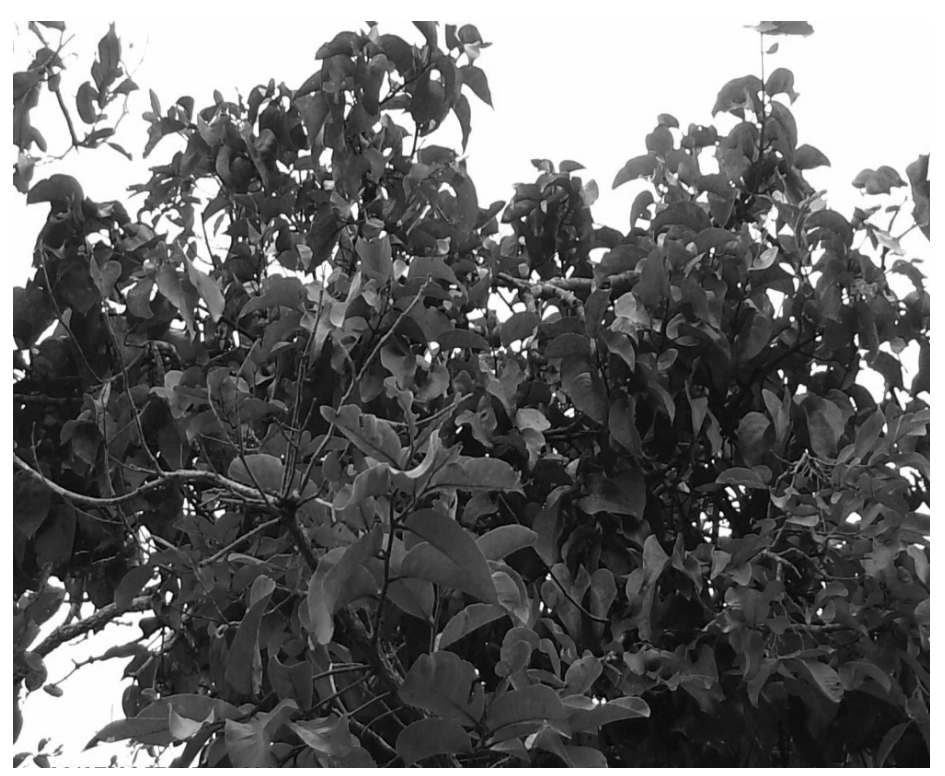

Figure 2: Rameaux feuillés de Tapinanthus bangwensis parasite des arbres et arbustes dans le département de Katiola.

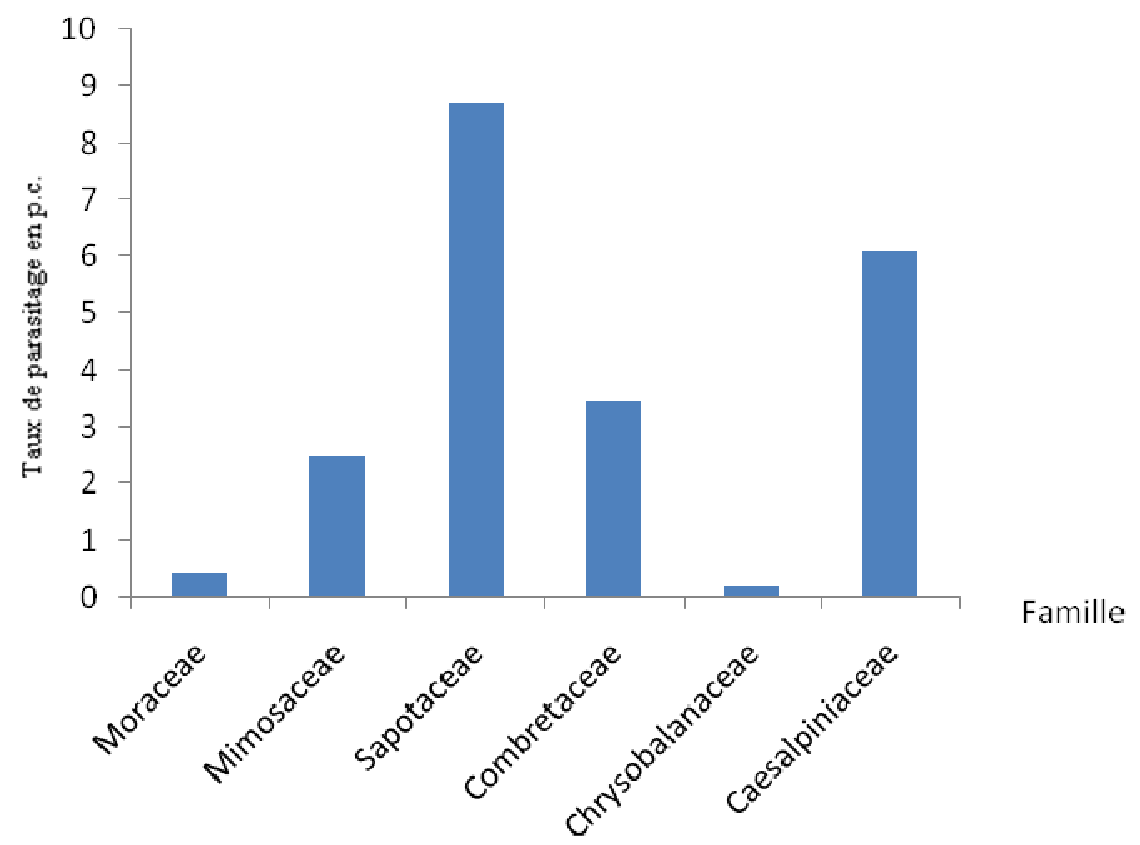

Figure 3 : Taux de parasitage des familles attaquées par les Loranthaceae. 
Tableau 1: Taux de parasitage des arbres et arbustes par les Loranthaceae dans le département de Katiola.

\begin{tabular}{lcc}
\hline & Taux de parasitage (p.c.) & Signification \\
\hline Arbre & 6,33 & $\mathrm{X}^{2}(1)=0,065$ \\
Arbuste & 3,51 & Pas de différence significative \\
Arbre-Arbuste & 5,47 & $\mathrm{P}>5 \%$ \\
\hline
\end{tabular}

\section{DISCUSSION}

Deux espèces de Loranthaceae ont été inventoriées sur les arbres et arbustes non cultivés dans le département de Katiola au Nord de la Côte d'Ivoire, ce sont Tapinanthus bangwensis (Engl. et K. Krause) Danser et Agelanthus dodoneifolius (D.C.) Polh et Wiens. Ce nombre est inférieur à celui obtenu par Traoré et al. (2003) dans le nord du pays. Cette faible quantité d'espèce de Loranthaceae obtenu peut être du au fait que notre étude n'a été réalisée que dans le département de Katiola contrairement à Traoré et al. (2003) dont les études ont été réalisées sur quatre départements.

Notons que l'espèce Tapinanthus bangwensis est une espèce ubiquiste qui n'est pas spécifique d'une végétation donnée et conviendrait à toutes les variations écologiques. Cette espèce a été observé aussi bien en zone forestière qu'en zone de savane préforestière (Soro et al., 2004 ; Soro, 2010). En effet, Soro (2010) a inventorié, en zone forestière, 5 espèces de Loranthaceae dont Tapinanthus bangwensis sur les arbres et arbustes dans les plantations de l'Ouest de la Côte d'Ivoire.

Le spectre d'hôtes de Agelanthus dodoneifolius (5 espèces hôtes) est plus large que celui de Tapinanthus bangwensis (1 espèce hôte). Ceci pourrait s'expliquer par le type de végétation. En effet, la savane préforestière est moins boisée que la zone forestière. Nos résultats diffèrent de Ceux de Amon (2006) et de Soro (2010). Le premier auteur a noté la présence de Tapinanthus bangwensis sur 96 espèces hôtes dans l'ouest de la Côte d'Ivoire. Tandis que pour le second, Tapinanthus bangwensis s'est retrouvé sur 57 espèces hôtes.
Les taux de parasitage enregistrés au cours de cette étude sont de 6,33 p.c. pour les arbres et 3,51 p.c. pour les arbustes spontanés. Concernant les plantations de culture pérenne, aucune espèce de Loranthaceae n'a été observée. La non infestation des cultures pérennes pourrait se justifier par le faible taux de parasitage des arbres et arbustes de la végétation naturelle. En effet, Amon (2006) signale que les taux de parasitage varient entre 1,02 p.c. et 65,58 p.c. pour les arbres et arbustes dans le département de Grandbassam. Soro (2010) note également que les cultures principales sont moins infestées que les arbres et arbustes spontanés dans les départements de Gagnoa, Soubré et Oumé.

Le faible taux de parasitage obtenu sur le site d'étude pourrait s'expliquer par le type de végétation du département qui est caractérisé par une dispersion et un nombre moins élevé d'arbre et d'arbuste défavorisant ainsi la dissémination des Loranthaceae. En effet, selon certains auteurs (Boussim, 2002), la dissémination des Loranthaceae se fait par des oiseaux qui se nourrissent de la viscine (pulpe collante) de leurs fruits. Après consommation de la pulpe, les graines rejetées restent fixées sur une branche de l'arbre hôte. Très souvent, les graines restent collées sur le bec de l'oiseau, par la viscine. Elles sont ainsi transportées et déposées d'arbre en arbre et donnent naissance à de nouveaux plants de parasite. Les Loranthaceae n'auraient donc pas une large gamme d'espèces à infester sur notre zone d'étude compte tenu de la dispersion et du nombre moins élevé d'arbre et d'arbuste sur la zone d'étude.

Les déformations observées sur les plantes hôtes au niveau des points d'insertion 
des parasites seraient la conséquence de la réaction des arbres à la contamination (Boussim, 2002). L'hôte réagit par la mise en place d'une boursouflure au niveau de la zone en contact indiquant ainsi que les connexions entre les tissus conducteurs des deux partenaires ont eu lieu (Engone Obiang, 2006).

La localisation préférentielle des Loranthaceae à la périphérie du houppier de leur hôte pourrait s'expliquer par un besoin de lumière chez le parasite. En effet, les Loranthaceae sont hémiparasites; elles puisent les éléments minéraux dans l'hôte grâce à leur suçoir, mais pour la synthèse des substances organiques, ces parasites usent de leurs propriétés chlorophylliennes. D'où la localisation à la périphérie des houppiers pour recevoir la lumière. De plus, des études botaniques indiquent que les espèces de Loranthaceae d'Afrique ont besoin de la lumière pour germer (Boussim, 2002).

$\mathrm{Au}$ cours de cette étude, aucune méthode de lutte n'est pratiquée par les populations locales dans le département de Katiola. La non présence des Loranthaceae sur les cultures pérennes et l'utilisation des Loranthaceae par les populations locales dans la pharmacopée pourrait justifier cette absence de méthode de lutte.

\section{Conclusion}

L'étude des Loranthaceae, plantes vasculaires parasites dans le département de Katiola, a permis d'identifier deux espèces des plantes épiphytes. Il s'agit de Agelanthus dodoneifolius et Tapinanthus bangwensis. Il ressort de cette étude que le taux de parasitage est de 6,33 p.c. pour les arbres et de 3,51 p.c. pour les arbustes. Les Loranthaceae sont localisées en général à l'extrémité du houppier. Malgré quelques perturbations causées par les Loranthaceae, aucune méthode de lutte n'est pratiquée par les populations locales dans le département de Katiola. Donc, une sensibilisation auprès des populations quant au rôle néfaste de ces hémiparasites sur les arbres et les arbustes. Aussi, une meilleure connaissance de la biologie de ces hémiparasites permettrait d'élaborer des stratégies de lutte efficace contre leur distribution dans les vergers et sur les plantes ligneuses spontanées. Il serait alors important d'étendre cette étude à tout le Nord et si possible à toute la Côte d'Ivoire, afin de mieux cerner le phénomène de parasitisme des Loranthaceae, en vue d'entreprendre des programmes de recherche et de méthodes de lutte efficaces contre ces parasites.

\section{REFERENCES}

Amon ADE, Soro D, N'guessan K, Traoré D. 2010. Les Loranthaceae: plantes vasculaires parasites des arbres et arbustes au sud-est de la Côte d'Ivoire. $J$. Appl. Biosc., 25: 1565 - 1572.

Amon ADE. 2006. Les plantes vasculaires parasites de la famille des Loranthaceae rencontrées dans le Département de Grand-Bassam, au Sud de la Côte d'Ivoire. Mémoire de D.E.A. de Botanique, Université de Cocody, U.F.R. Biosciences. Abidjan, Côte d'Ivoire, 57 p.

Baillon F, Frochot H. 1987. La Lutte chimique contre le gui des feuillus. Pénétration et distribution dans le gui de deux herbicides à absorption foliaire. Phytoma, Défense des Cultures, 391: 1621.

Boussim IJ. 2002. Les Phanérogames parasites du Burkina-faso: inventaire, taxonomie, écologie, et quelques aspects de leur biologie. Cas particulier des Loranthaceae parasites du karité. Thèse de doctorat d'état ès Sciences naturelles. $285 \mathrm{p}$.

Boussim IJ, Nayéré M. 2009.Méthodes de lutte contre les Loranthaceae. Publication Server of Goethe University, 12. 27-35.

Boussim IJ, Sallé G, Guinko S. 1993. Tapinanthus parasite du karité au Burkina faso. Bois et Forêts des Tropiques 238: 45-64.

Engone Obiang NL, Sallé G. 2006. Faut-il éradiquer Phragmanthera capitata, 
parasite des hévéas ? C.R. Biologies, 329: 185-195.

Frochot H, Sallé G. 1980. Modalités de dissémination et d'implantation du gui. R.F.F.XXXII, 6: 505-518.

Habiyaremye FX. 1997. Etude phytosociologique de la dorsale orientale du lac Kivu. Musée Royal de l'Afrique Centrale, Tervuren, Belgique. Annales Sciences Economiques, 24: 276.

Hevecam (hévéa au Cameroun). 1995. Rapport de la direction exploitation agricole : bilan de l'essai Loranthus12AGRO/DEA, 9 p.

Ganglo CJ. 1999. Poster presented at the Proceedings of the XVIth International Botanical Congress. August 1-7, 1999, Saint Louis, Missouri, USA. 531 p.

Guillaumet JL, Adjanohoun E. 1971. Le milieu naturel de la Côte d'Ivoire. La végétation. Mémoires O.R.S.T.O.M. Adiopodoumé, 158-232.

Koffi AA. 2004. Evaluation de l'incidence des Loranthaceae sur la productivité de Hevea brasiliensis (Kunth) Mull.Arg. à Anguédédou (Sud de la Côte d'Ivoire) Mémoire de DEA de Botanique, Université de Cocody, Abidjan, 52 p.

Robbins K, Johnson DW, Hawksworth FG \& Nicholls TH. 1996. Aerial application of ethephon is ineffective in controlling logepole pine dwarf misteletoe. In Dwarf mistletoe: Biology, Pathology, Systematics, hawksworth F, Wiens D. (eds). United State Departement of Agriculture Forest Service, Agricultural Handbook: $410 \mathrm{p}$.

Sallé G, Frochot H, Andary C. 1993. Le gui. La Recherche, 260: 1334-1342.

Sinsin G. 1993. Phytosociologie, écologie, valeur pastorale et production et capacité de charges des pâturages du périmètre nikki-kalalé au Nord-Bénin. Th. Doct. Fac. Sc., Lab. Bot. Syst. \& Phyt., Uni. Lib. Bruxelles, 390 p.

Sokpon N. 1995. Recherches écologiques sur la forêt dense semi-décidue de pobè au sud- est du Bénin: Groupements végétaux, structure, régénération et chute de litière. Th. Doct. Fac. Sc., Lab. Bot., Syst. \& Phyt., Uni. Lib. Bruxelles, 350 p.

Soro D, N'da-Adopo A, Da KP, Traoré D. 2004. Lutte contre les parasites chez le karité. Agronomie Africaine, 16(3): 2128.

Soro D, Ouattarra D, Da KP, Traoré D. 2004. Efficacité de l'émondage contre les Loranthaceae ou Guis du Karité : cas du parc naturel à karités de Tengrela, dans le Nord de la Côte d'Ivoire. Annales de Botanique Afrique de l'Ouest, 03: 87-95.

Soro K. 2010. Les Loranthaceae ou guis, plantes vasculaires parasites des arbres et arbustes cultivés ou non, dans l'ouest de la Côte d'ivoire: cas des départements d'Oumé, de Gagnoa et de Soubré. Thèse Unique d'Écologie Végétale, option Agroforestérie, Université de Cocody, Abidjan, 189 p.

Traoré D, Da KP. 1996. Lutte contre les plantes vasculaires du karité et du néré dans le nord de la Côte d'ivoire. Cas des départements de Korhogo, Boundiali, Ferkéssédougou et Tengrela. Rapport annuel du P.E.P A.I.S.A. -CI- 97 p.

Traoré D, Da K P, Soro D. 2003. Lutte contre les plantes vasculaires parasites du Karité, dans le Nord de la Côte d'Ivoire. Cas du parc naturel à karités de Tengrela. Université de Cocody. U.F.R. Biosciences. Laboratoire de Botanique. Rapport du P.E.P. A.I.S.A.-CI, 116 p. 